\title{
Editorial
}

\section{The Next Battleground for Patient Safety: Influenza Immunization of Healthcare Workers}

\author{
Christopher J. Hoffmann, MD, MPH; Trish M. Perl, MD, MSc
}

\section{The farther backward you look, the farther forward you are likely to see.-Winston Churchill}

In 1918, more people died of influenza in 24 days than in 24 years of the human immunodeficiency virus epidemic, including many healthcare workers (HCWs) and influenza researchers. ${ }^{1}$ Today, although we may not be in the midst of a global pandemic, we have significant numbers of healthcare-associated influenza infection in addition to community-acquired infections. For example, last year, a 37-yearold man with a history of complex congenital heart disease was transferred, on a ventilator, to our institution because of respiratory failure thought to be due to Eisenmenger's syndrome. His evaluation for possible heart-lung transplantation revealed influenza, likely acquired at the outside institution. The patient avoided an unnecessary operation but was one of many healthcare-associated episodes of influenza that occurred last winter in U.S. hospitals. The tragedy of this and similar episodes worldwide is that many influenza infections could be prevented by vaccinating $\mathrm{HCW}$ s. Fortunately, our patient recovered from his intensive care ordeal and was not added to the estimated 36,000 , or 1 in 10,000 , Americans who die annually of influenza. ${ }^{2,3}$ A new approach to implementation of HCW vaccination is needed, achieved through effective programming, determined local leadership to champion programs, and institutional support to enable infection control leaders to partner with their employee health colleagues to implement successful programs.

$\mathrm{HCW}$ vaccination reduces patient mortality and is part of the Centers for Disease Control and Prevention's strategy for prevention and control of influenza. ${ }^{4,5}$ As the percentage of vaccinated $\mathrm{HCWs}$ increases, healthcare-associated influenza decreases. ${ }^{6}$ Vaccination of HCWs decreases influenza infection among $\mathrm{HCWs}$ by $88 \%^{7}$ and reduces patient mortality by $50 \% .4,7$, Finally, vaccination improves clinical safety by decreasing workplace disruption from absenteeism. ${ }^{7,9}$ Despite these compelling data, fewer than half of the HCWs in the United States receive the influenza vaccine each year. ${ }^{5}$

A year ago, Infection Control and Hospital Epidemiology published an editorial and articles describing effective interventions and significant strides individual institutions have made in improving $\mathrm{HCW}$ vaccination rates. ${ }^{10,11}$ This issue of Infection Control and Hospital Epidemiology includes further studies examining personal and institutional barriers to achieving higher rates of $\mathrm{HCW}$ vaccination. Studies in this issue by Wodi et al. and Tapiainen et al. continue to build the knowledge base for building a successful vaccine delivery infrastructure. Specifically, they identify individual $\mathrm{HCW}$ barriers to vaccination. ${ }^{12,13}$ Wodi et al. focused on resident physicians in a single hospital; Tapiainen et al. evaluated a broad range of HCWs in a single children's hospital. Both groups showed that the primary reason for $\mathrm{HCW}$ s to receive influenza vaccination was to protect themselves, not patients. Among resident physicians, Wodi et al. reported the most common reason for not getting vaccinated was lack of time (47\%). Remarkably, $31 \%$ of all respondents indicated that influenza vaccination could cause influenza, suggesting a clear need for further education. Tapiainen et al. examined barriers to influenza vaccination in a variety of HCWs-nurses, physicians, and ancillary staff-and the impact of an educational intervention designed to overcome identified barriers

The authors are from the Division of Infectious Diseases, Department of Medicine, Johns Hopkins University School of Medicine, Baltimore, Mary land. Dr. Perl is also from the Department of Hospital Epidemiology and Infection Control, The Johns Hopkins Hospital, Baltimore, Maryland.

Address correspondence to Trish M. Perl, MD, MSc, 425 Osler, 600 North Wolfe Street, The Johns Hopkins Hospital, Baltimore, MD 21287. tperl@jhmi.edu 
in their children's hospital. After an educational intervention, vaccination rates rose from $43 \%$ to $64 \%$ among physicians. Perhaps their most important finding was that the primary reason for getting vaccinated shifted from self-protection to protection of patients after the educational intervention.

These two studies present several important messages. First, they point to a continued inadequate understanding of influenza among $\mathrm{HCWs}$, especially regarding the patient safety benefit of $\mathrm{HCW}$ vaccination and the potential side effects from influenza vaccination. Second, they indicate that convenience should not be overlooked in $\mathrm{HCW}$ vaccination programs. Third, shifting the message from self-interest to altruism in protecting patients may improve vaccination rates. Taken together, they provide further support for taking vaccination beyond the realm of basic employee health, making it a patient safety issue. $\mathrm{HCW}$ vaccination can be viewed as a means of protecting patients from influenza exposure and the related mortality seen among vulnerable populations and should be presented as such to both HCWs and the hospital leadership. This is in contrast to a vaccination strategy and message aimed at reducing worker absenteeism (although also important). Asking HCWs to stay home if they have influenza-like symptoms is impractical because half of those with influenza infection have mild symptoms or no symptoms at all. ${ }^{14}$ The second way that vaccination should move to the patient care setting is by physically providing $\mathrm{HCW}$ vaccination services in wards, clinics, and common meeting areas, such as outside the hospital cafeteria. In addition to the emphasis on the patient safety message, moving vaccination services to patient care areas helps to overcome inconvenience and the time spent going to an employee health clinic or influenza vaccination station (lack of time is the main reason cited for foregoing vaccination). These recommendations are not new. Successful influenza vaccine programs have shown that taking the vaccine to healthcare's trenches (ie, the wards and clinics) using mobile cart programs and implementing mass vaccination after conferences and grand rounds have improved vaccination compliance. ${ }^{6,15}$

We have reached a point of having sufficient research to design programs that overcome individual barriers and increase $\mathrm{HCW}$ influenza vaccination. Unfortunately, despite development of creative strategies, repeated calls from the hospital infection control community, and isolated examples of success, we continue to suffer nationally from unacceptably low rates of HCW vaccination. This policy issue competes for attention with many other important issues; more understanding alone is not enough to overcome institutional inertia. The major challenge now is capturing the attention of hospital administrators to gain institutional support needed to implement necessary programs. Until this happens, the work from studies published in this issue, and in others before and after it, will not reach the full potential of decreasing morbidity from healthcare-associated influenza by increasing $\mathrm{HCW}$ vaccination.

Providing a reward to institutions for achieving high levels of HCW vaccination will bring necessary institutional attention to vaccination. This can be effectively accomplished by national organizations, such as the Society for Healthcare
Epidemiology of America, the Infectious Diseases Society of America, and the Association for Professionals in Infection Control and Epidemiology, taking a position on requiring mandatory vaccination of $\mathrm{HCW}$. In addition, making $\mathrm{HCW}$ vaccination a quality measure of the Joint Commission on Accreditation of Healthcare Organizations (JCAHO) and the Centers for Medicare \& Medicaid Services (CMS) or a process measure to report to the public as proposed by the Centers for Disease Control and Prevention's Healthcare Infection Control Practices Advisory Committee ${ }^{16}$ would enhance these efforts. A good place to start is subsuming $\mathrm{HCW}$ influenza vaccination under the safety goal of reducing healthcare-associated infections on the JCAHO and CMS report card. This should be attractive to the JCAHO and the CMS, as they are looking for quantifiable interventions with a clear evidence-based benefit to expand their hospital evaluation mechanisms. This will enable leaders in healthcare epidemiology and infection control and employee health to garner needed attention and support from hospital administrators to move forward with applying the knowledge we have to build effective $\mathrm{HCW}$ vaccination programs. Among the myriad of reasons to vaccinate $\mathrm{HCWs}$, there are few, if any, down sides. It is time to move the fight to the wards, healthcare epidemiology and infection control societies, and national regulatory agencies.

\section{REFERENCES}

1. Barry JM. The Great Influenza: The Epic Story of the Deadliest Plague in History. London: Penguin Books; 2004.

2. Thompson WW, Shay DK, Weintraub E, et al. Mortality associated with influenza and respiratory syncytial virus in the United States. JAMA 2003;289:179-186.

3. Poland GA, Tosh P, Jacobson RM. Requiring influenza vaccination for health care workers: seven truths we must accept. Vaccine 2005;23:2251-2255.

4. Carmen WF, Elder AG, Wallace $L A$, et al. Effects of influenza vaccination of health-care workers on mortality of elderly people in long-term care. Lancet 2000;355:93-97.

5. Harper SA, Fukuda K, Uyeki TM, et al. Prevention and control of influenza. $M M W R$ 2005;54 (RR-08):1-40.

6. Salgado CD, Giannetta ET, Hayden FG, et al. Preventing nosocomial influenza by improving the vaccine acceptance rate of clinicians. Infect Control Hosp Epidemiol 2004;25:923-928.

7. Wilde JA, McMillan JA, Ser wint J, et al. Effectiveness of influenza vaccine in health care professionals. JAMA 1999;281:908-913.

8. Potter J, Stott DJ, Roberts MA, et al. Influenza vaccination of health care workers in long-term-care hospitals reduces the mortality of elderly patients. I Infect Dis 1997;175:1-6.

9. Saxen H, Virtanen M. Randomized, placebo-controlled double blind study on the efficacy of influenza immunization on absenteeism of health care workers. Pediatr Infect Dis J 1999;18:779-783.

10. Wu HM, Abrutyn E. Influenza and pneumococcal vaccination: how far we have come and how to go farther. Infect Control Hosp Epidemiol 2004;25:901-903.

11. Kimura AC, Higa JI, Nguyen C, et al. Interventions to increase influenza vaccination of healthcare workers. $M M W R$ 2005;54:196-199.

12. Wodi AP, Samy S, Ezeanolue E, et al. Influenza vaccine: immunization rates, knowledge, and attitudes of resident physicians in an urban teaching hospital. Infect Control Hosp Epidemiol 2005;26:867-873.

13. Tapiainen T, Bär G, Schaad UB, Heininger U. Influenza vaccination among healthcare workers in a university children's hospital. Infect Control Hosp Epidemial 2005;26:855-858.

14. Elder AG, O'Donnell B, McCruden EA, et al. Incidence and recall of influenza in a cohort of Glasgow healthcare workers during the 1993-4 epidemic: results of serum testing and questionnaire. BMJ 1996;313:1241-1242.

15. Sartor C, Tissot-Dupont H, Zandotti C, et al. Use of a mobile cart influenza program for vaccination of hospital employees. Infect Control Hosp Epidemiol 2004;25:918-922.

16. McKibben L, Horan T, Tokars JI, et al. Guidance on public reporting of healthcare-associated infections: recommendations of the Healthcare Infection Control Practices Advisory Committee. Infect Control Hosp Epidemiol 2005;26:580-587. 